\title{
Effects of distal mutations on ligand-binding affinity in $E$. coli Dihydrofolate reductase
}

Chen-Hua Huang ${ }^{2 \dagger}$, Yun-Wen Chen ${ }^{2 \dagger}$, Tsun-Tsao Huang ${ }^{2}$, Ya-Ting Kao ${ }^{* 1,2,3}$

${ }^{1}$ Department of Biological Science and Technology, National Yang Ming Chiao Tung University, Hsinchu, Taiwan 30068, ROC.

${ }^{2}$ Institute of Bioinformatics and Systems Biology, National Yang Ming Chiao Tung University,

Hsinchu, Taiwan 30068, ROC.

${ }^{3}$ Center For Intelligent Drug Systems and Smart Bio-devices (IDS2B), National Yang Ming Chiao Tung University, Hsinchu, Taiwan 30068, ROC.

*Corresponding author e-mail: yatingkao@nycu.edu.tw (Ya-Ting Kao)

${ }^{\dagger}$ C.-H. H. and Y.-W. C. contributed equally to this work. 


\section{SUPPORTING INFORMATION}

Four supplemental figures: Figure S1 Figure S5.

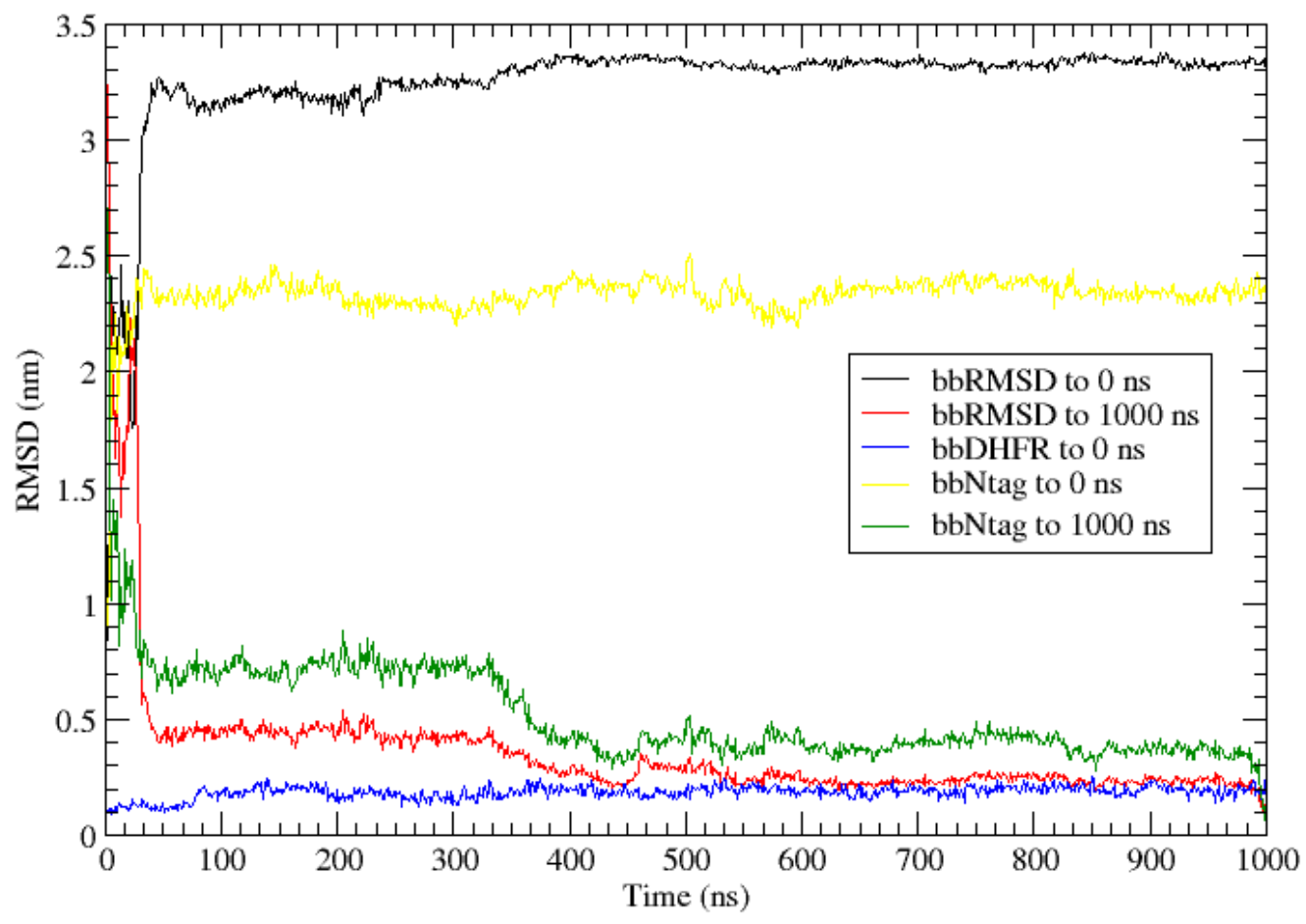

Figure S1. The root-mean-square deviation of the backbone (bbRMSD) for the $1000 \mathrm{~ns}$ MD run with respect to the reference conformation at 0 ns (black line) and the conformation at $1000 \mathrm{~ns}$ (red line). The blue line is the backbone RMSD for the DHFR part, showing that the bbRMSD is about 1-2 $\AA$ during the simulation and indicating that the DHFR part of the tagDHFR was stable during the 1000 ns simulation. On the other hand, the backbone RMSD for the N-terminus-tag was shown in yellow line (the reference conformation at $0 \mathrm{~ns}$ ) and green line (the reference conformation at $1000 \mathrm{~ns}$ ), suggesting that $\mathrm{N}$-terminus-tag conformation was stable in the equilibration portion of the trajectory ( $>600 \mathrm{~ns})$. 

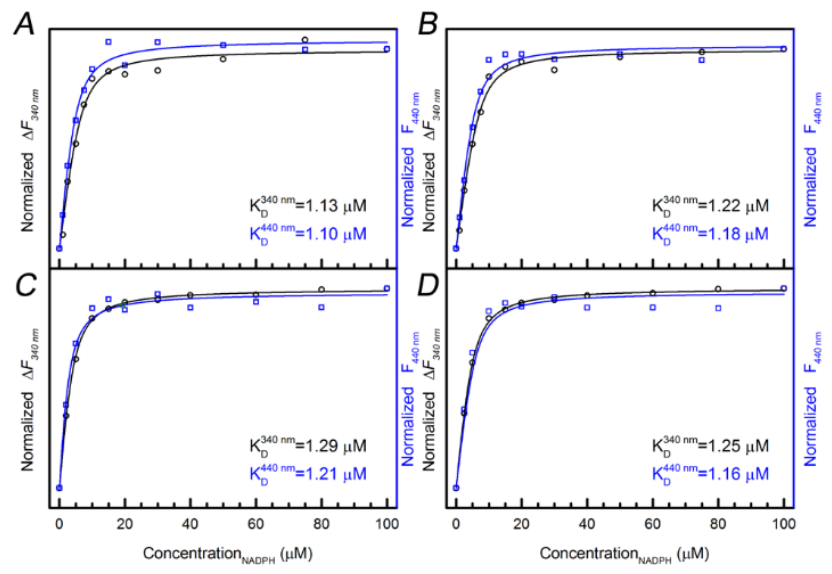

Figure S2. The comparison between $\Delta F_{340 \mathrm{~nm}}$ and $F_{440 \mathrm{~nm}}$ analyses in wildtype DHFR. (A D) Four individual trials of wildtype DHFR binding with NADPH under the same conditions with two analysis methods $\left(\Delta F_{340} \mathrm{~nm}\right.$ and $F_{440 \mathrm{~nm}}$ analyses). The results from the two analysis methods are consistent. The black circle and black fitting line are from $\Delta F_{340 \mathrm{~nm}}$ analysis. The blue square and blue fitting line are from $F_{440 \mathrm{~nm}}$ analysis. For clarity comparison, both curves are normalized at the NADPH concentration of $100 \mu \mathrm{M}$. 

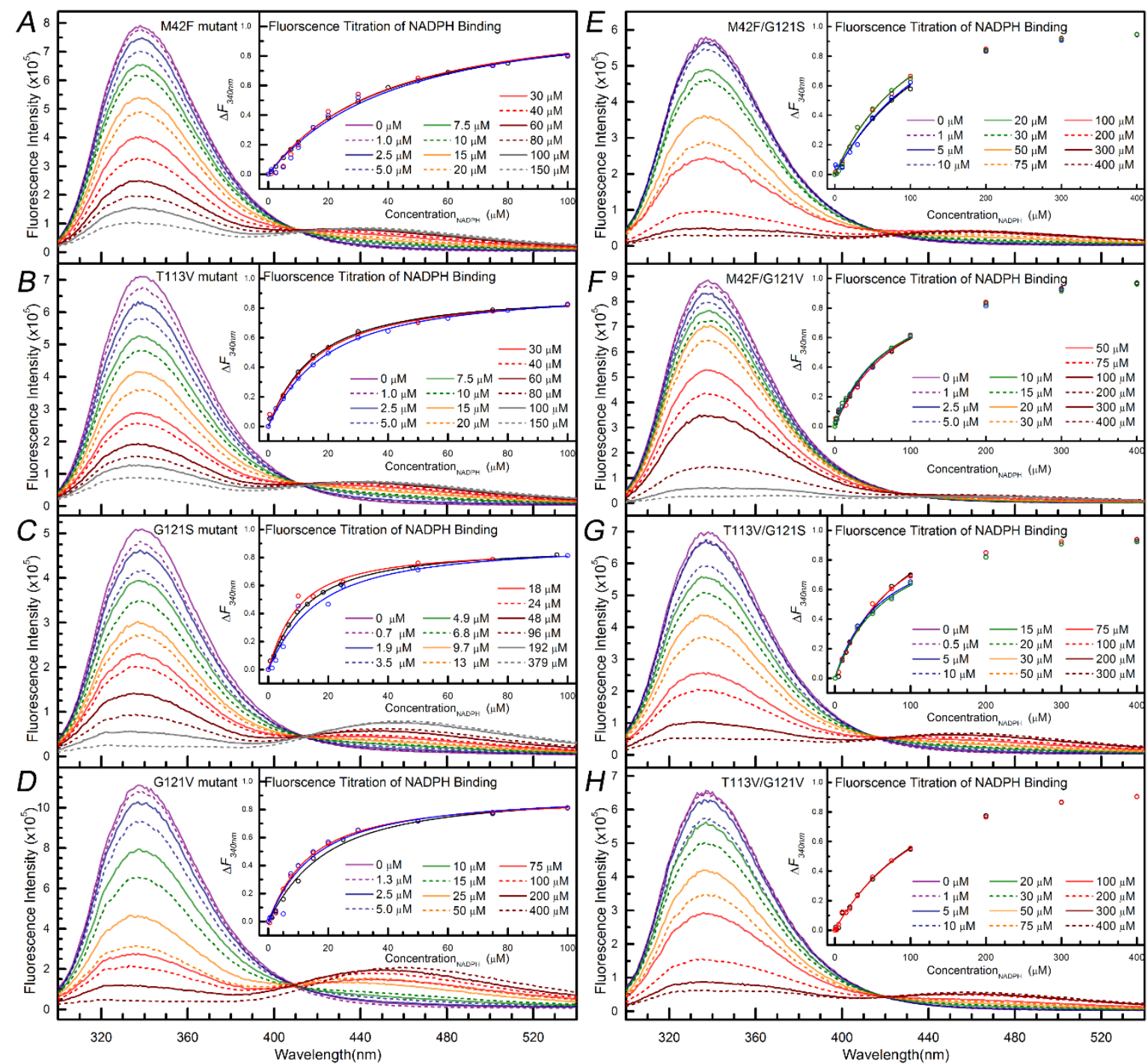

Figure S3. The cofactor-binding titration experiments on (A D) single-point mutation and $(\mathrm{E} \sim \mathrm{H})$ double-point mutation. With the increasing concentration of NADPH, the decrease at 340-nm fluorescence and the increase at 440-nm fluorescence were observed. Inset shows the $\mathrm{K}_{\mathrm{D}}$ analysis curves and the range of $\Delta F_{340 \mathrm{~nm}}$ is from -0.1 to 1.1. All $\mathrm{K}_{\mathrm{D}}$ constants from data analysis up to $100 \mu \mathrm{M}$ NADPH are listed in Table 1. 

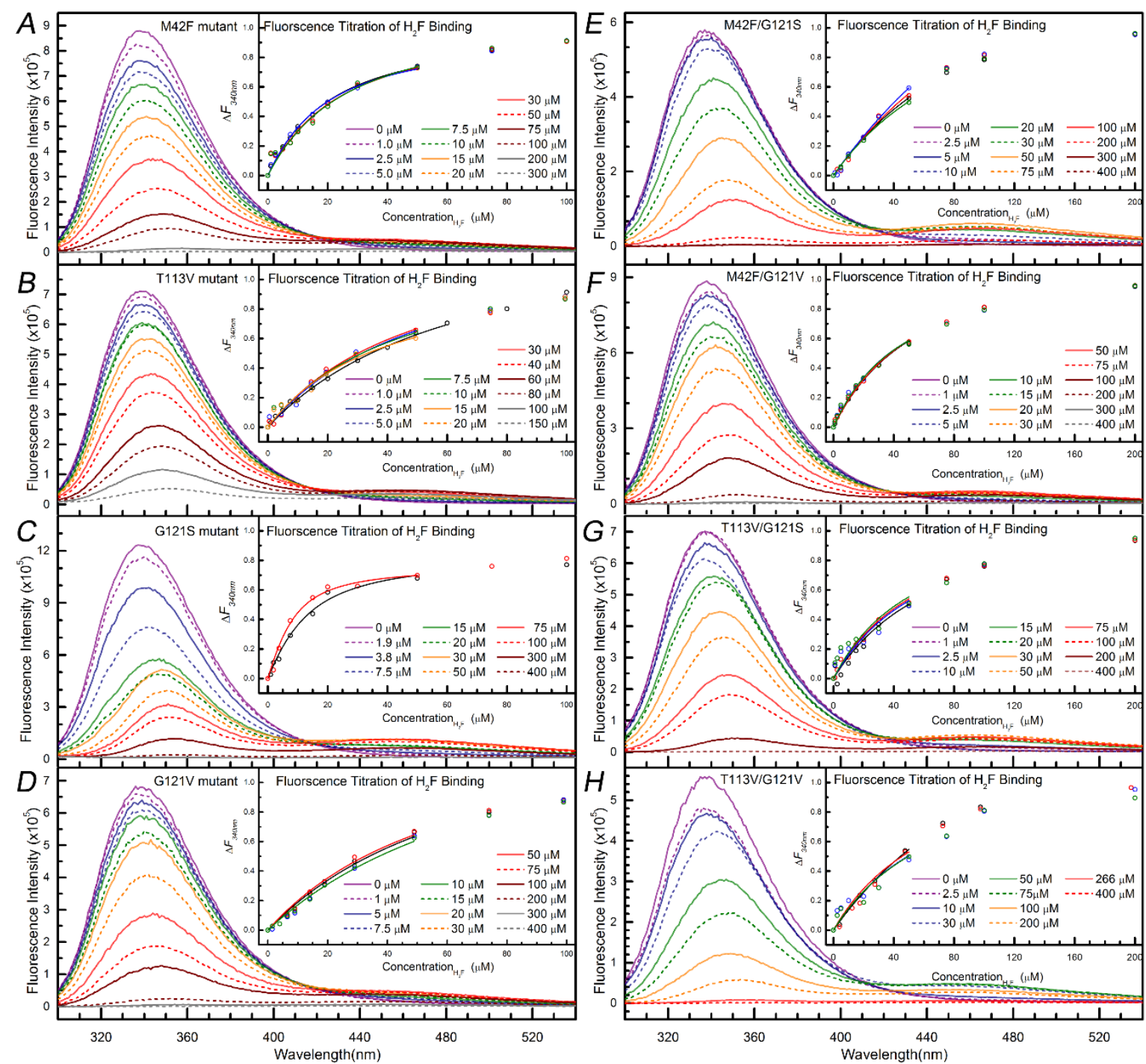

Figure S4. The substrate-binding titration experiments on $(\mathrm{A} \sim \mathrm{D})$ single-point mutation and $(\mathrm{E} \sim \mathrm{H})$ double-point mutation. With the increasing concentration of $\mathrm{H}_{2} \mathrm{~F}$, a decrease at 340$\mathrm{nm}$ fluorescence was observed. Based on the $\mathrm{H}_{2} \mathrm{~F}$-deduction methods described in Text and Figure 3. Inset shows the $\mathrm{K}_{\mathrm{D}}$ analysis curves and the range of $\Delta F_{340 \mathrm{~nm}}$ is from -0.1 to 1.1 .

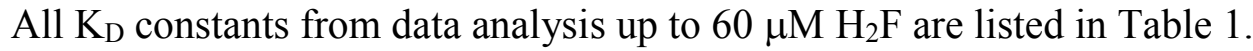




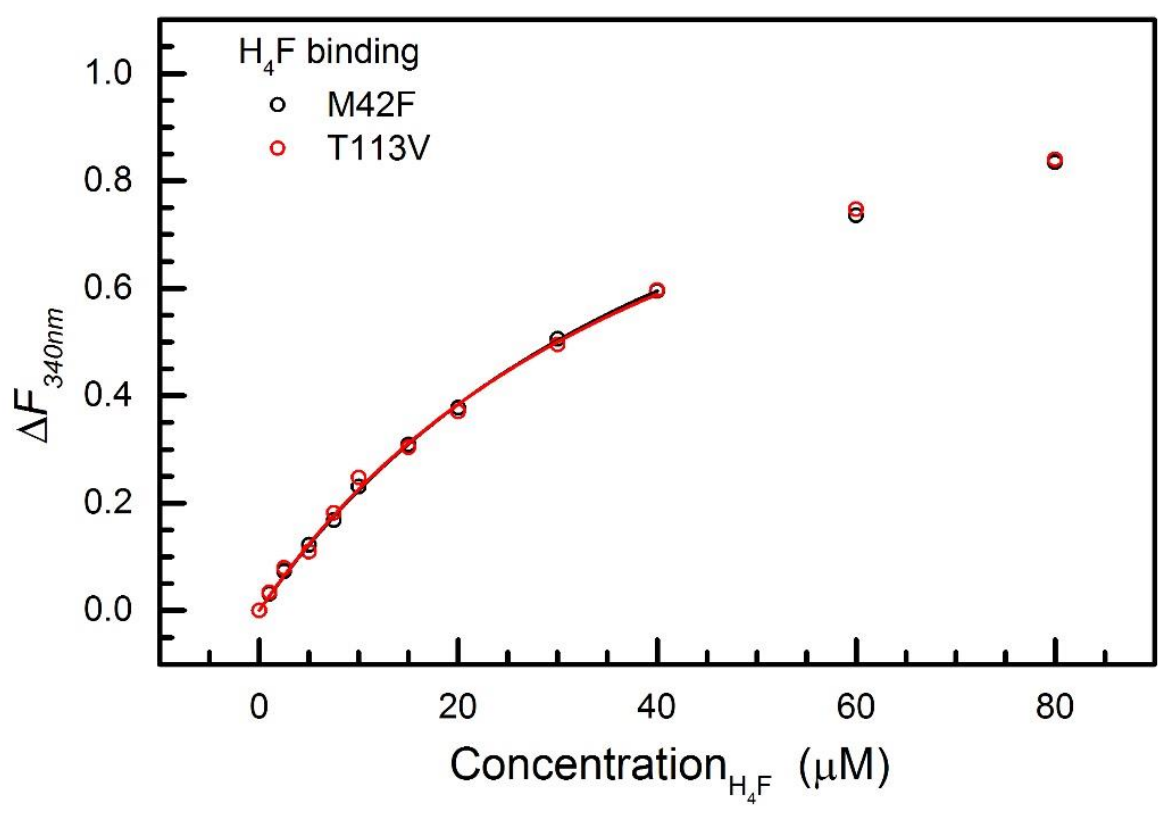

Figure S5. The $\mathrm{K}_{\mathrm{D}}$ analysis curves of M42F (black circle) and T113V (red circle). Based on the $\mathrm{H}_{4} \mathrm{~F}$-deduction methods described in Text and Figure5, the enzyme-product dissociation constants of M42F-ecDHFR and T113V-ecDHFR were estimated as $44.01 \pm 2.73 \mu \mathrm{M}$ and $40.87 \pm 2.23 \mu \mathrm{M}$, respectively. 